\title{
Image Processing for Art Investigation
}

\author{
Bruno Cornelis \\ Department of Electronics and Informatics (ETRO), Pleinlaan 2, B-1050 Brussels, Belgium \\ Advisors: Ann Dooms, Ingrid Daubechies \\ Date and location of PhD thesis defense: 13 October 2014, Vrije Universiteit Brussel
}

Received 24th February 2015; accepted 27th July 2015

\begin{abstract}
Computer processing of digital images of paintings has become a fast growing and challenging field of research during the last few years. Our contribution to this research field consists of a set of tools that are based on dimensionality reduction, sparse representations and dictionary learning. These tools are used to assist in art related matters such as restoration, conservation, art history, material and structure characterization, authentication, dating and even style analysis. Since paintings are complex structures the analysis of all pictorial layers and their support requires a multimodal set of high-resolution image acquisitions.

The presented research can broadly be subdivided into three large categories. The first one is the removal of artifacts present in high-resolution acquisitions of paintings, caused by the painting's support. In case of canvas paintings, the natural texture of the canvas may change over time by various external factors and become very prominent. We propose two methods for the cleaning of images from canvas artifacts to improve their visual reading by art experts. The first method is based on Wiener filtering and an extension with a lowcomplexity spatially adaptive module to tackle non-stationarity of the data [1]. The second method approaches canvas removal as a source separation problem where the image is modeled as the superposition of two distinct components - the canvas and the painting - with very different morphologies. We use the idea of sparse representations to separate the two components of the image with the only condition that the dictionaries that are used to sparsely represent each component are mutually incoherent (Figure 1). We also investigated the removal of cradling artifacts in X-ray images of panel paintings. A three-step approach was proposed [2]; first the cradled areas are detected and a uniform luminance inconsistency caused by the cradling is removed. In a second step the texture, present in the cradled areas, is separated from the actual painting. Finally, a refinement step learns the distinction between the texture belonging to the cradle and that from the panel.

The second main field of research is automated crack detection within the famous Ghent Altarpiece, which is meant to help in the delicate matter of its conservation but also as guidance during its ongoing restoration. Depending on the data that was at our disposal we proposed two different detection schemes; the method in [3] operates on a single image modality (i.e. visible photography) and combines by means of a voting scheme three crack detection techniques: oriented elongated filters, a multiscale extension of the morphological top-hat transformation and a detection method based on dictionary learning. In 2012 a high resolution and multimodal dataset of the Ghent Altarpiece was made available to the larger public, which allowed for new crack detection
\end{abstract}

Correspondence to: <bcorneli@etro.vub.ac.be >

Recommended for acceptance by Jorge Bernal

DOI http://dx.doi.org/10.5565/rev/elcvia.715

ELCVIA ISSN:1577-5097

Published by Computer Vision Center / Universitat Autònoma de Barcelona, Barcelona, Spain 

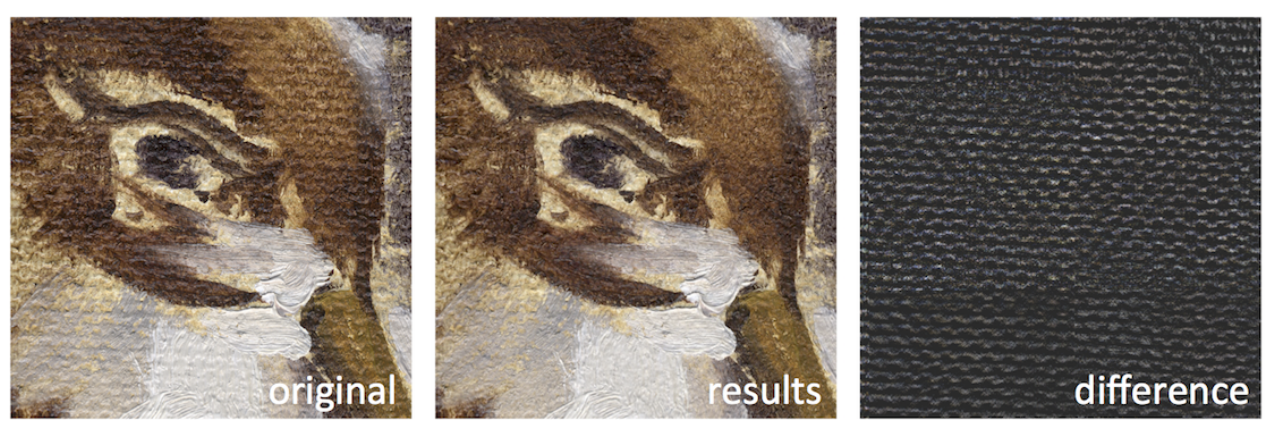

Figure 1: Example of canvas removal based on sparse representations.

techniques that are able to make use of the information provided by each modality and yielding a more reliable detection scheme. We adopted a Bayesian approach that estimates for each pixel a posterior probability of belonging to a crack, given a large set of feature vectors extracted over all modalities [4]. The Bayesian classification has the significant advantages of selecting the most important information from each modality and of keeping parameter tuning to a minimum. The produced crack maps can be used directly for the assessment of the state of conservation of the masterpiece but were also used as input for a patch-based inpainting algorithm that virtually restores the painting and helped in improving the legibility of a book in the Virgin Annunciate panel. Figure 2 depicts detection results of the first method and the Bayesian based detection on respectively the old and the 2012 dataset.
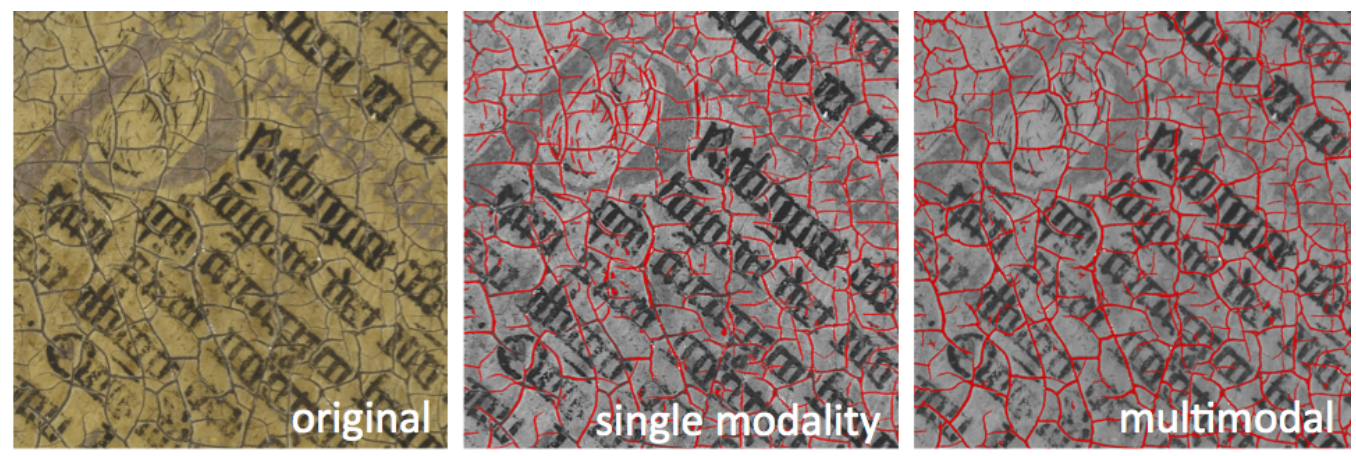

Figure 2: Crack detection results: (left) original image - (middle) using methods of [3] - (right) Bayesian method of [4].

Lastly I developed a set of art forensics methods that can be deployed in applications such as authentication and dating of paintings. In case of canvas paintings, X-ray images are typically analysed since canvas threads are unveiled in this particular imaging modality. We studied which features are distinguishing enough for determining the fingerprint of the canvas [5]. These features are then used to firstly deduce the type of canvas, but also to determine the origin of a piece of canvas, which can prove handy when tying different paintings over time. One can imagine that when the canvas fingerprints of two paintings are linked to the same bolt of canvas, the paintings were most likely painted by the same artist/studio or at least during the same period. We also investigated the segmentation of multispectral images using Bayesian discriminant analysis. These segmentation results can be used as a preprocessing step for other applications such as crack and restoration detection. Lastly we investigated painted pearls, which are abundant in the Ghent Altarpiece. The visual appearance of these relatively simple objects can be modelled mathematically and new metrics that are closely related to the visual properties of pearls were proposed. The goal is to analyse the consistency of the painter throughout different parts of the masterpiece and compare how much the pearls differ from those of contemporaries. The initial experiments on a rather limited dataset show encouraging results [6]. 
The various contributions, briefly described above, are all detailed in my $\mathrm{PhD}$ thesis [7].

\section{References}

[1] B. Cornelis, A. Dooms, J. Cornelis, P. Schelkens, "Digital canvas removal in paintings", Signal Processing 92(4):1166-1171, 2012.

[2] R. Yin, D. Dunson, B. Cornelis, B. Brown, N. Ocon, I. Daubechies, "Digital Cradle Removal in X-ray Images of Art Paintings", IEEE International Conference on Image Processing, Paris, 2014.

[3] B. Cornelis, T. Ruzic, E. Gezels, A. Dooms, A. Pizurica, L. Platisa, J. Cornelis, M. Martens, M. De Mey, I. Daubechies, "Crack detection and inpainting for virtual restoration of paintings: The case of the Ghent Altarpiece", Signal Processing 93(3): 605-619, 2013.

[4] B. Cornelis, Y. Yang, J. T. Vogelstein, A. Dooms, I. Daubechies, D. Dunson, "Bayesian crack detection in ultra high resolution multimodal images of paintings", arXiv preprint arXiv:1304.5894, 2013.

[5] B. Cornelis, A. Dooms, A. Munteanu, J. Cornelis, P. Schelkens, "Experimental study of canvas characterization for paintings", Proc. of IS\&T/SPIE Electronic Imaging 2010, San Jose, CA, USA, 2010.

[6] L. Platisa, B. Cornelis, T. Ruzic, A. Pizurica, A. Dooms, M. Martens, M. De Mey, I. Daubechies, "Spatiogram features to characterize pearls in paintings", IEEE International Conference on Image Processing, Brussels, Belgium, 2011.

[7] Bruno Cornelis, Image Processing for Art Investigation, University Press, ISBN: 978-9-4619722-5-5, October 2014. http://bmkitmf.etro.vub.ac.be/Pub_Files/Phd/PhD_Bruno_Cornelis.pdf 